Abstract

\title{
Investigation of Insect Resistance Components in Wild Pigeonpea Cajanus Scarabaeoides ${ }^{\dagger}$
}

\author{
Thi My Linh Hoang *, Abigail Dawit, Brett Williams, TJ Higgins and Sagadevan Mundree \\ Centre for Agriculture and the Bioeconomy, Queensland University of Technology, Brisbane 4001, Australia; \\ a.dawit@hdr.qut.edu.au (A.D.); b.williams@qut.edu.au (B.W.); tj.higgins@csiro.au (T.H.); \\ sagadevan.mundree@qut.edu.au (S.M.) \\ * Correspondence: 1t.hoang@qut.edu.au; Tel.: +61731380617 \\ + Presented at the third International Tropical Agriculture Conference (TROPAG 2019), Brisbane, Australia, \\ 11-13 November 2019.
}

Published: 31 December 2019

\begin{abstract}
Cajanus scarabaeoides (L.) Thouars is the closest wild relative of cultivated pigeonpea, Cajanus cajan (L.) Millspaugh. However, unlike cultivated pigeonpea which is very susceptible to insects, especially Helicoverpa armigera (Hubner), this wild pigeonpea is strongly insect resistant. Since $H$. armigera causes damage to many important crops resulting in economic losses up to 2 billion USD/year, improvement in resistance to this insect in crops is highly desirable. Here we investigate insect resistance components in C. scarabaeoides and explore the possibility of transferring one or more of those factors to cultivated pigeonpea. A detached leaf assay was used to assess antibiosis and antixenosis resistance mechanisms in C. scarabaeoides. Artificial diet supplemented with lyophilised leaf powder was employed to investigate the antibiosis resistance mechanism. Data on larval mortality and larval and pupal weights were collected. Time taken for neonate larvae to pupate and for pupae to develop to the moth stage were determined through daily observations. Preliminary results showed that $H$. armigera larval weight was significantly reduced and larval development stages were prolonged when reared on different accessions of $C$. scarabaeoides as compared to the susceptible check, C. cajan (ICPL 87). Interspecific hybridization was carried out between $C$. scarabaeoides and a commercial pigeonpea variety. Results from insect challenge assays and trichome identification indicate that the hybrids have similar levels of insect resistance to their wild parent. Proteomic analysis is being used to identify possible antibiosis factors.
\end{abstract}

Keywords: cajanus cajan; cajanus scarabaeoides; pigeonpea; helicoverpa armigera; antibiosis; antixenosis; trichome

Funding: This project was funded by AssociatedGrain (scholarship) and Advance Queensland Research Fellowship AQRF14816-17RD2.

Acknowledgments: The authors thank QUT-CARF for the technical support and Australian Grains Gene bank (Horsham, Victoria, Australia) for providing the seeds used in the study.

Conflicts of Interest: The authors declare no conflict of interest.

(C) 2019 by the authors. Licensee MDPI, Basel, Switzerland. This article is an open access article distributed under the terms and conditions of the Creative Commons Attribution (CC BY) license (http://creativecommons.org/licenses/by/4.0/). 